\title{
EL DISEÑO DE UN CURRÍCULO MULTINIVEL A PARTIR DE LA AUTOPERCEPCIÓN DEL ALUMNADO DE MAGISTERIO: UNA MIRADA EMPODERADORA
}

\author{
María Pilar Rodrigo Moriche \\ Universidad Autónoma de Madrid
}

\begin{abstract}
RESUMEN: El trabajo que aquí se presenta parte de un proyecto de innovación donde un equipo interdepartamental de docentes de la Facultad de Formación de Profesorado y Educación de la Universidad Autónoma de Madrid se planteó la incorporación de la perspectiva multinivel en sus aulas a través de una propuesta metodológica donde los estudiantes fuesen agentes de la educación inclusiva. En este artículo, de manera concreta, se presenta el proceso de investigación llevado a cabo en el marco de la asignatura Didáctica General, donde se establecieron como objetivos principales favorecer la toma de consciencia del alumnado sobre la diversidad en sus estilos de aprendizaje, y adecuar la metodología docente según los criterios del currículo multinivel. A partir de una metodología exploratoria y aplicada se buscó un diseño curricular que permitiese personalizar el aprendizaje. Para ello se pasaron dos cuestionarios a 133 alumnos del Grado de Magisterio que permitió conocer su autopercepción, su nivel de empoderamiento, y su estilo y preferencias de aprendizaje. Como resultado se presenta una propuesta pedagógica con enfoque multinivel que pretende ser un referente empoderador capaz de propiciar aprendizajes coherentes entre la teoría y la práctica docente universitaria desde un planteamiento de inclusión de máximos.
\end{abstract}

PALABRAS CLAVE: Empoderamiento, inclusión, innovación docente, currículo multinivel, Magisterio.

\section{MULTI-LEVEL CURRICULUM DESIGN BASED ON TEACHER TRAINING STUDENTS' SELF-PERCEPTION: AN EMPOWERMENT PERSPECTIVE}

\footnotetext{
ABSTRACT: The work presented here derives from an innovation project where an inter-departmental team of teachers at the Teacher Training Faculty of the Universidad Autónoma de Madrid in which an inter-departmental
} 
team of teachers at the Teacher Training Faculty proposed the incorporation of a multi-level approach in their classrooms through a methodological proposal where the students were agents of inclusive education. In this article, more concretely, under the framework of General Didactics the principal aims were set to favour the students, awareness of diversity in their learning styles, and to adapt the Teaching Guide to multi-level curriculum criteria. From a baseline of exploratory and applied methods, the target was to design a curriculum that would allow learning to be personalised according to each student's individual. The two questionnaires given to 133 students in Teacher Training Degree has allowed us to gather knowledge of students' self-perceived, level of empowerment and their learning style and preferences. The result is a proposal for a curriculum with a multilevel approach intended as a reference for empowerment propitiating coherent learning from the theory and practice of university teaching from an approach of inclusion of maximums.

KEYWORDS: Empowerment, inclusion, teaching innovation, multi-level curriculum, teacher training.

Recibido: $12 / 12 / 2018$

Aceptado: 17/06/2019

Correspondencia: María Pilar Rodrigo Moriche, Facultad de Formación del Profesorado y Educación, C/ Francisco Tomás y Valiente, 3, 28049 Madrid. Email: pilar.rodrigo@uam.es.

\section{INTRODUCCIÓN}

De la Torre (2009) apunta como problemática de la enseñanza universitaria la descontextualización con la realidad, donde se prioriza el conocimiento teórico sobre el práctico; en respuesta Oliver (2009) aboga por la organización del conocimiento en asignaturas como el medio para no sólo permitir el traspaso de informaciones y de conocimientos, sino como un marco referencial para el conocimiento personal e intransferible que cada alumno construye desde la experiencia vivencial que le aporte la propia asignatura. En la misma línea Toro (2010) resalta el valor de lo cotidiano, y de manera expresa la importancia de la cotidianeidad como potencial de un aprendizaje consistente para los futuros maestros, aspecto clave y necesario desde que se inicia la andadura universitaria en la carrera de Magisterio.

La pluralidad del alumnado requiere de variedad de objetivos, metodologías, e incluso de evaluaciones. El currículo multinivel (Collicott, 1991, 2000) se plantea como una opción para atender a la diversidad de capacidades y permite al maestro planificar para todo el alumnado en una misma clase; pero este no comienza con el diseño de una programación de aula para un grupo de infantil, primaria o secundaria, sino que empieza con una mirada y una intencionalidad: la docente. Una mirada que hay que cultivar y orientar en el docente novel, ya que la experien- 
cia vivencial en su formación inicial va a subyacer en la construcción de su perfil profesional.

Díez y Sánchez (2015) toman los referentes normativos para la atención a la diversidad en el ámbito universitario -Ley 51/2003, la LOU y el RD 1393/2007para mostrar la no discriminación e igualdad de oportunidades mediante medidas de acción positiva. En la misma línea el reciente Informe sobre el estado de la Educación Inclusiva en España (Comité de la Organización de Naciones Unidas, 2018) expone que un sistema de educación inclusiva debe fundamentarse en una modalidad para todos los estudiantes donde se atienda a lo que cada alumno requiera. De este modo se atenderá a una inclusión de máximos que va a permitir que cada alumno interaccione desde su singularidad presente.

Se justifica así el diseño del currículo multinivel como una estrategia capaz de romper las barreras participativas; y como señala Arnáiz (1999) donde se atiende a la diversidad al posibilitar que cada alumno encuentre actividades acordes a su nivel de competencia curricular. Porque tal y como fundamenta Rodrigo-Moriche (2018) nos encontramos en un modelo basado en los derechos humanos donde "todo individuo tiene capacidades que han de potenciarse, y discapacidades, de carácter permanente o transitorio, que será necesario compensar a lo largo de su trayectoria vital mediante diseños que se adecuen a sus características reales" (p. 35).

En conexión con el currículo multinivel, el Diseño Universal de Aprendizaje (DUA) determina la intención y la sistematicidad desde el inicio, lo que va a favorecer atender a las diferencias individuales (CAST, 2008); se conforma como un reto ético y sobre todo creativo en relación a la planificación, diseño, gestión y administración (Rodrigo-Moriche, 2018). El marco del DUA está sustentado por sus tres principios fundamentales que conectan con el currículo multinivel: múltiples formas de representación de la información para facilitar su comprensión, diferentes medios para la acción y la expresión según los perfiles individuales del alumnado, y diversas formas de participación que permiten aumentar la implicación y la motivación del alumnado.

De manera complementaria a este proceso inclusivo se hace necesario recoger el concepto de empoderamiento juvenil (Soler, Trilla, Jiménez-Morales y Úcar, 2017) definido en base a un modelo pedagógico donde se determinan espacios, momentos y procesos de empoderamiento desarrollados en el Proyecto Hebe ${ }^{1}$, y donde destacan las posibilidades de decisión, actuación, participación e intervención en aquello que le afecta -al joven- siendo responsable con la colectividad. Uno de los cuatro espacios más referenciados en este proyecto por los jóvenes en la adquisición de su empoderamiento fue el espacio formativo (Planas, Trilla, Garriaga, Alonso y Monseny, 2016) por lo tanto cualquier docente universitario que quiera aportar al empoderamiento de los jóvenes estudiantes (y en el caso que nos ocupa a futuros docentes) deberá en primer lugar conocer sus capacidades, diseñar acciones que promuevan

1. Proyecto HEBE: El empoderamiento de los jóvenes: análisis de los momentos, espacios y procesos que contribuyen al empoderamiento juvenil. Ref. EDU2013-42979-R. http://www.projectehebe.com/es/ sobre-projecte-hebe/. 
la toma de decisiones y potenciar sus actuaciones en coherencia con las decisiones adoptadas.

Todo lo anterior cobra esencia no solamente con programaciones multinivel y empoderadoras, sino también conscientes, donde modelos pedagógicos renovados conectan con la realidad cuando se produce un cambio de consciencia individual, pero también grupal, ya que "la meta de una educación futura no es alcanzar la cima del conocimiento, sino alcanzar una consciencia evolucionada y enfocada en el mundo en el que vivimos. No se trata de una ética y consciencia individuales sino colectivas." (De la Torre, 2009, p. 11). Este cambio se produce acompañado de nuevas actitudes, creencias y valores interiorizados en interacción con los otros y en un medio favorable, lo que conforma nuevos estilos de vida del individuo y de la sociedad.

\section{Método}

\section{Planteamiento del problema}

El problema que evidencia esta investigación es la desconexión entre teoría y práctica en el ámbito universitario y la falta de adaptación curricular a los perfiles de aprendizaje del alumnado de una manera individualizada. Se puntualiza en las siguientes cuestiones:

- iEs posible proporcionar al alumnado universitario novel de Magisterio una herramienta que le permita realizar una toma de consciencia sobre la autopercepción de su empoderamiento, y sus estilos y preferencias de aprendizaje?

- ¿Se puede diseñar una propuesta pedagógica y curricular de la materia Didáctica General que se adapte a los perfiles individuales y grupales del alumnado del primer curso de Magisterio Infantil y Primaria?

\section{Objetivos}

De manera concreta los objetivos de esta investigación son:

(1) Tomar consciencia por parte del alumnado de su autopercepción, su empoderamiento y sus estilos de aprendizaje individuales y grupales.

(2) Adecuar la propuesta pedagógica y curricular de la asignatura Didáctica General a los perfiles individuales y grupales recogidos atendiendo a los criterios del currículo multinivel.

\section{Diseño de la investigación}

Para dar respuesta a las preguntas de esta investigación se procedió a diseñar una metodología exploratoria y aplicada centrada en la práctica educativa, que pretendía emplear el conocimiento de la actividad cotidiana para obtener un primer contacto con la realidad y presentar el diseño de una propuesta didáctica con perspectiva multinivel en el ámbito universitario. 
Partimos por tanto de un estudio cualitativo y cuantitativo cuyas claves del éxito residen en la aplicación de técnicas de aprendizaje activo, el papel participativo del estudiante, y la reflexión y propuesta de mejora del proceso educativo por parte del docente.

\section{Contexto y participantes}

La muestra objeto de este estudio está formada por dos grupos de estudiantes de $1^{\circ}$ de Magisterio de los Grados de Primaria, y Doble Grado de Infantil y Primaria, que cursan la asignatura anual Didáctica General (6 ECTS) formado por un total de 133 alumnos. Se detallan las características concretas de cada uno de los grupos en la tabla 1.

Tabla 1. Características de la muestra

\begin{tabular}{lcccccccc}
\hline \multicolumn{1}{c}{ Especialidad } & $\begin{array}{c}\mathbf{N}^{\mathbf{0}} \\
\text { alumnos/as } \\
\text { totales }\end{array}$ & Chicas & Chicos & \multicolumn{4}{c}{ Edades } \\
\hline Primaria & 65 & 36 & 29 & 0 & 55 & 9 & 1 \\
Infantil y Primaria & 68 & 64 & 4 & 2 & 63 & 2 & 1 \\
\hline TOTAL & 133 & 100 & 33 & & & & \\
\hline
\end{tabular}

El perfil del alumnado es ligeramente diferente en ambos grupos, algunos aspectos a tener presentes son que al ser la nota de corte para el acceso a la carrera de Doble Grado casi de dos puntos por encima de la nota de Primaria nos encontramos con un perfil más autoexigente académicamente; por otro lado, mientras que en Primaria el género masculino y femenino del alumnado se aproxima, en Doble Grado el porcentaje masculino es muy bajo; por último, otro elemento descriptivo que distingue a ambos grupos es que hay un perfil más físico-deportivo en Primaria que en Doble Grado.

\section{Recogida de información: instrumentos y procedimientos}

El análisis de datos se realizó a través de dos cuestionarios: un cuestionario durante la primera semana del curso centrado en la trayectoria personal y profesional del alumnado, donde se recogían datos cualitativos (tabla 2) que nos ofrecían un conocimiento previo tanto a nivel individual como grupal del alumnado sobre:

- Aspectos personales y profesionales

- Concepciones en relación a la Educación en general, y del Grado en particular.

- Autopercepción y valoración sobre el trabajo en equipo. 
Tabla 2. Preguntas Cuestionario 1. Trayectoria personal y profesional

\begin{tabular}{ll}
\hline \multicolumn{1}{c}{ Dimensiones } & \multicolumn{1}{c}{ Preguntas } \\
¿Cómo soy? & Tus puntos fuertes/Tus puntos débiles \\
& Cualidades/Habilidades personales e interpersonales \\
& Señala el valor más importante de tu vida (uno solo) ¿Por qué? \\
Experiencias de mi & Describe brevemente tu trayectoria vital (biografía) \\
vida & Con qué sueñas en el futuro \\
& En qué te gusta emplear tu tiempo libre \\
& Tu otro currículo (qué sé hacer, qué he aprendido en otros campos) \\
& Indica cualquier otro aspecto que consideres de interés \\
& ¿Qué te interesa profesionalmente de la Titulación de Grado? ¿Por qué? \\
Mi desarrollo & ¿Has trabajado o trabajas en algo relacionado con este campo? En caso \\
profesional & afirmativo describe qué labor desempeñas o has desempeñado. \\
& ¿Qué cosas te interesan profesionalmente? \\
& ¿Qué entiendes por Educación? \\
& ¿Tienes otra formación relacionada con la educación (monitor, entre- \\
& nador...)? ¿Qué conoces sobre la Educación Primaria? \\
& ¿Qué decisiones has tomado respecto a tu presente o futuro profesional? \\
& ¿Qué planes te has planteado para poder conseguirlos? \\
& ¿Qué cualidades tienes para el trabajo en equipo? \\
& ¿Qué ventajas e inconvenientes consideras que conlleva el trabajo en \\
& equipo? \\
& ¿Qué requisitos consideras que hay que tener individualmente para \\
trabajar en equipo? & ¿Qué valoración le das al trabajo en equipo en el desarrollo de tu tarea \\
profesional? & Concluye indicando lo que te ha aportado esta reflexión sobre ti mismo/a \\
& y si te ha permitido detectar algunos puntos fuertes y/o débiles. \\
\hline Trabajo en equipo final & \\
& \\
&
\end{tabular}

Y un segundo cuestionario (tabla 3), tras dos meses iniciado el curso, que aportó datos cuantitativos en relación al aprendizaje multinivel, elaborado a partir de:

- la medida y cuantificación de las dimensiones que determinan el empoderamiento juvenil (Úcar, Planas, Novella y Rodrigo-Moriche, 2017);

- los estilos y preferencias de aprendizaje a partir de las inteligencias múltiples de Gardner (1995), y los sistemas alternativos de comunicación (Paredes, Esteban y Rodrigo-Moriche, 2018);

- los estilos de aprendizaje y las preferencias de agrupación para el trabajo de tareas según Collicot (1991);

- las actividades preferidas a partir del cuestionario Honey-Alonso de estilos de aprendizaje (Alonso, Gallego y Honey, 2010);

- y, los roles de equipo según Belbin (2005). 
Tabla 3. Preguntas Cuestionario 2. Aprendizaje multinivel

\begin{tabular}{|c|c|}
\hline Bloques & Preguntas \\
\hline $\begin{array}{l}\text { Bloque I. } \\
\text { Autopercepción de } \\
\text { empoderamiento }\end{array}$ & $\begin{array}{l}\text { Marca el nivel de adquisición personal que tienes de cada uno de } \\
\text { estas dimensiones, siendo 1: Muy Bajo y } 4 \text { : Muy alto } \\
\text { Dimensiones de empoderamiento: autoestima, autonomía, capa- } \\
\text { cidad crítica, eficacia, identidad comunitaria, meta-aprendizaje, } \\
\text { participación, responsabilidad y trabajo en equipo. }\end{array}$ \\
\hline $\begin{array}{l}\text { Bloque II. } \\
\text { Estilo de } \\
\text { aprendizaje }\end{array}$ & $\begin{array}{l}\text { Señala el grado en que estas inteligencias te identifican para ad- } \\
\text { quirir el aprendizaje. } \\
\text { Inteligencias: lingüística, lógica-matemática, visual/espacial, físi- } \\
\text { co/cinestésica, intrapersonal e interpersonal. } \\
\text { Escala de valoración: } 1 \text { : Nada; y } 4 \text { : Totalmente } \\
\text { Indica tus preferencias comunicativas para la lección de clase. } \\
\text { Sistemas alternativos de comunicación: visual, auditiva, audiovi- } \\
\text { sual, cinestésica, otras. }\end{array}$ \\
\hline $\begin{array}{l}\text { Bloque III. Trabajo } \\
\text { en equipo }\end{array}$ & $\begin{array}{l}\text { Ordena según tu preferencia de trabajo. } \\
\text { Opciones: individual, en parejas, pequeños grupos y gran grupo. } \\
\text { En relación a tu trabajo en equipo, señala los roles que sueles } \\
\text { desempeñar: } \\
\text { Roles: investigadora de recursos, cohesionadora, coordinadora, } \\
\text { cerebro, monitora evaluadora, especialista, impulsora, implemen- } \\
\text { tadora y/o finalizadora. } \\
\text { Escala de valoración: Nunca; Alguna vez; Casi siempre; y Siempre. }\end{array}$ \\
\hline $\begin{array}{l}\text { Bloque IV. } \\
\text { Actividades }\end{array}$ & $\begin{array}{l}\text { Mis actividades favoritas... } \\
\text { Opciones: las que plantean como reto hacer algo nuevo; aque- } \\
\text { Ilas que están muy bien estructuradas y se puede seguir las ins- } \\
\text { trucciones de manera clara; aquellas que permiten experimentar } \\
\text { y practicar las últimas técnicas y novedades; y aquellas otras en } \\
\text { las que se dispone de tiempo para preparar el trabajo y realizarlo } \\
\text { a conciencia. }\end{array}$ \\
\hline $\begin{array}{l}\text { Bloque V. } \\
\text { Valoración final }\end{array}$ & $\begin{array}{l}\text { ¿Crees que esta tarea te ha aportado un mayor conocimiento sobre } \\
\text { ti mismo/a y tu estilo de aprendizaje? } \\
\text { Opciones: Sí/No/Otras }\end{array}$ \\
\hline
\end{tabular}

La pretensión de ambos cuestionarios, además de obtener los datos cualitativos y cuantitativos para el diseño de una propuesta didáctica con enfoque multinivel, era favorecer que el alumnado tomara consciencia a través de su cumplimentación, de la importancia de sus trayectorias personales y profesionales, su nivel de empoderamiento y, su estilo y sus preferencias de aprendizaje.

Los cuestionarios se han elaborado mediante la herramienta de formularios de google y se les ha facilitado el enlace a través de la plataforma de la asignatura. El procedimiento que se ha llevado a cabo ha sido en primer lugar pasar un consentimiento informado en formato papel, donde se les ponía en conocimiento del 
tratamiento y uso de los datos que iban a proporcionar y la consiguiente autorización para su uso en esta investigación. Aunque los datos personales aparecen en ambos cuestionarios - pues forman parte de una tarea calificable de la asignatura- el tratamiento de los datos ha sido codificado para anonimizar la información recogida.

\section{Resultados}

\section{Trayectoria personal y profesional}

Los resultados de este cuestionario arrojan luz sobre la autoconciencia del proceso de vida y de aprendizaje que aporta la propia experiencia del alumnado como punto de partida para iniciar su proceso formativo como maestros. Se convierte en un "darse cuenta de" (De la Torre, 2009):

La verdad es que me he sorprendido a mí misma pensando en algo tan sencillo como mis gustos o preferencias; nunca lo había hecho de esta manera y pienso que debería hacerlo más a menudo para explotar mis puntos fuertes y mejorar los que no lo son. (Tarea de Trayectoria personal y profesional, 2018)

Poner por escrito tantas cosas sobre mi vida, mis cualidades y mis esperanzas sobre esta carrera y el futuro profesional me ha ayudado a ordenar mis ideas y a plantearme estos años como el método de llegar a la meta que quiero, utilizando todas mis habilidades y puntos fuertes. (Tarea de Trayectoria personal y profesional, 2018)

Aparecen aspectos que refuerzan la cotidianeidad (Díez, 2009). Los conocimientos pasados adquiridos mediante las experiencias se convierten en referentes donde cimentar y proyectar los conocimientos futuros que van a adquirir durante este primer curso:

De la educación infantil y de primaria, conozco lo que he ayudado a mi hermano de 8 años, viendo el tipo de examen que le hacen y el temario que le corresponde por su edad. Aparte de a los niños que he dado clase, de distintas edades. Y por último tengo familiares y gente conocida que me ha hablado de esta carrera, por supuesto dejando claro que no es como bastante gente la ve de sencilla. (Tarea de Trayectoria personal y profesional, 2018)

La percepción que tienen los estudiantes de la trayectoria personal y profesional se aprecia de manera integrada, donde toda experiencia pasada configura la experiencia presente, siendo un fiel reflejo de lo que supone la teoría de la complejidad de Morín (2004) cuando cita la frase de Pascal "tengo por imposible concebir las partes al margen del conocimiento del todo, tanto como conocer el todo sin conocer particularmente las partes".

A los dos años de nacer, mis padres se separaron, desde pequeña he sido scout donde tengo mi grupo de amigos más cercanos y voy a danza desde los 3 años. Al cumplir mi hermana mayor 13 años tuvimos que elegir donde vivir y lo pase muy mal porque mis 
padres no se llevan bien. Y durante mi etapa en el instituto he tenido alguna depresión unas más grandes que otras. Sueño con formar una familia, poder trabajar de profesora, vivir en Madrid y mantener todas mis amistades de hoy en día. Por las noches me gusta leer, también me gusta mucho escuchar música y bailar, salir con mis amigos, jugar con mi hermana pequeña y de vez en cuando hacer voluntariados. (Tarea de Trayectoria personal y profesional, 2018)

Tras la revisión de las tareas individuales, se mostró al grupo-clase la información a nivel grupal mediante una presentación de las trayectorias personales y profesionales que las caracteriza como grupo. La percepción que obtienen tras esta presentación es grupal: una nueva toma de consciencia de las potencialidades que tienen como grupo.

\section{Aprendizaje multinivel}

Los resultados de la tarea de aprendizaje multinivel orientan el diseño de la propuesta pedagógica, y dan respuesta al segundo objetivo de esta investigación: adecuar la propuesta pedagógica y curricular de la asignatura Didáctica General a los perfiles individuales y grupales recogidos atendiendo a los criterios del currículo multinivel.

La percepción de los niveles de empoderamiento del 80\% de la muestra de ambos grupos es alto o muy alto. Tan solo la dimensión de autoestima en el grupo de Doble Grado es valorado como bajo y muy bajo por un 34\%; y en el grupo de Primaria la dimensión de participación se posiciona en las puntuaciones más bajas (33\%).

En cuanto a los estilos de aprendizaje difiere en ambos grupos: en el grupo de Doble Grado los estilos de aprendizaje se caracterizan por las inteligencias de tipo interpersonal, seguido de intrapersonal y lingüística; en Primaria las inteligencias más aceptadas son la intrapersonal y la físico-cinestésica. En ambos grupos la inteligencia con menor grado de aceptación es la lógico-matemática.

Las preferencias comunicativas son similares para ambos grupos. Muestran preferencia en torno al 50\% hacia estilos de comunicación cinestésicos; en segundo lugar, de tipo visual, seguido de audiovisual. Siendo un porcentaje menor al 10\% quienes eligen sistemas comunicativos auditivos. Esto nos sitúa en un planteamiento de actividades alejadas de las clases magistrales.

En cuanto a su preferencia de agrupación para la realización de trabajos en equipo, hay diferencias en ambos grupos. En el grupo de Doble Grado se prioriza el trabajo individual frente al de equipo; mientras que el grupo de Primaria da preferencia al trabajo en pequeños grupos. En ambos casos prefieren en segundo lugar el trabajo por parejas. En tercer lugar, vuelven a diferir, y el alumnado de Doble Grado prefiere trabajar en pequeños grupos mientras que el grupo de Primaria prefiere de manera individual. Ambos señalan en último lugar el trabajo en gran grupo, estructura vinculada también a las clases magistrales.

Mayoritariamente en ambos grupos prefieren actividades en las que disponen de tiempo para preparar el trabajo y realizarlo a conciencia, siendo más elegidas 
este tipo en el grupo de Doble Grado que en Primaria. A continuación hay un interés por aquellas actividades que plantean como reto hacer algo nuevo, siendo mayor el interés que muestra el alumnado de Primaria. El alumnado de Doble Grado $(26,5 \%)$ requiere de actividades muy bien estructuradas y que permitan seguir las instrucciones claras en un mayor grado que los alumnos de Primaria $(15,4 \%)$. Por último señalan las actividades en las que se puede experimentar y practicar las últimas técnicas y novedades, siendo en el caso de Doble Grado el interés menor al $10 \%$.

Los resultados sobre la toma de consciencia apuntan a que más de un $80 \%$ en ambos grupos señalan que la tarea les ha aportado un mayor conocimiento sobre sí mismos y su estilo de aprendizaje lo que viene a dar una respuesta afirmativa a la primera pregunta de esta investigación.

\section{Diseño de propuesta pedagógica y curricular}

Una vez extraídos los perfiles de ambos grupos, podemos presentar el diseño de la propuesta pedagógica que se ha llevado a cabo durante el curso escolar y que ha permitido vivenciar en el aula el enfoque multinivel, basado en las cuatro fases propuestas por Collicot (2000):

a) Identificación de los conceptos subyacentes de la materia. Se estima en primer lugar establecer cuáles serán los conceptos básicos que todo el alumnado deberá dominar para superar la materia, estos son: Objetivos - Contenidos Competencias - Metodología - Evaluación - Recursos - Organización didáctica - Niveles de concreción - Programación didáctica de aula - Innovación educativa.

Los conceptos señalados se consideran los mínimos denominadores para todos los alumnos.

b) Determinar el método de presentación docente. El diseño de la propuesta de currículo con enfoque multinivel se ha fundamentado en metodologías que potencian el empoderamiento de los estudiantes, y desarrollan sus diferentes inteligencias; además se han adaptado los métodos a sus estilos de aprendizaje y sus preferencias comunicativas. Como limitaciones nos hemos encontrado con el espacio del aula (tradicional con pizarra/ proyector, tarima, y mesas en filas y orientadas hacia la pizarra) que al ser compartida con otros docentes y cada uno con sus propias metodologías, se hace difícil transformar desde una cultura individualista en las que nos encontramos (Armengol, 1999); y por otro lado el elevado número de alumnos por aula (68/65). Estos aspectos han dificultado la flexibilidad metodológica y la atención a la individualidad. No obstante, y con los datos resultantes de los cuestionarios hemos Ilevado a la práctica un método de presentación docente orientado a estilos de comunicación cinestésicos, materializado principalmente por el movimiento libre en el aula, donde los alumnos podían moverse en su interacción con los demás mediante el trabajo en pequeños grupos; se han propuesto actividades 
visuales como presentaciones, esquemas, imágenes, mapas conceptuales; y audiovisuales, como vídeos explicativos de los conceptos, y de situaciones educativas prácticas. Se han evitado las clases meramente expositivas respondiendo así al bajo interés por el estilo auditivo. Aunque se han presentado actividades de todos los tipos, han primado las actividades planificadas y notificadas con tiempo suficiente para su realización por parte del alumnado, junto con un guión de orientaciones y señalados los plazos de entrega. También se han Ilevado a cabo actividades que presentaban algún reto (feria-expositiva, vídeo-expositivo) y otras de experimentación (programación de aula).

Sus preferencias por los agrupamientos nos ha llevado a distinguir la acción en cada uno de los grupos: en Primaria se ha priorizado una distribución de tareas en pequeños grupos, mientras que en Doble Grado se ha orientado a tareas individuales. Como alternativa se ha facilitado la posibilidad de trabajar en parejas, y por último se han realizado desdobles en el aula para reducir el número de alumnos en las clases de gran grupo.

Cabe señalar que en todo el proceso metodológico se ha flexibilizado con técnicas, estrategias e incluso ajustes temporales propuestos por parte del alumnado.

c) Determinar el método de práctica del alumno

Una de las conclusiones más evidentes de este estudio es la demanda por parte de ambos grupos de reducir al máximo las clases magistrales. Para ello se propuso una estructura de actividades que permitieran al alumnado adoptar un papel activo y participativo, ajustándose principalmente a los resultados de sus estilos y preferencias de aprendizaje.

Las actividades propuestas se han estructurado en actividades iniciales, de desarrollo y de evaluación. Las actividades iniciales por lo general están bien estructuradas y se pueden seguir las instrucciones de manera clara, y tienen como objetivo que el alumnado experimente con la materia (p. ej. tarea de trayectoria personal y profesional, y búsqueda de legislación y comparativa); las actividades de desarrollo se plantean como aquellas que van a dar un conocimiento más profundo de la materia y hay un tiempo suficiente para realizarlo a conciencia (p. ej. debate sobre temática actual relacionada con la materia, y la programación de aula); finalmente las actividades de evaluación por lo general plantean algún reto que les permite hacer algo nuevo (examen grupal, feria-exposición y vídeo-exposición).

En la tabla 4 se expone el listado de actividades, y en la tabla 5 la relación de cada actividad con los aspectos de empoderamiento considerados más necesarios para los grupos según los resultados obtenidos (A1: Autoestima; A2: Participación), los estilos de aprendizaje (B1: I. lingüística; B2: I. interpersonal; B3: I. intrapersonal; B4 I. físico-cinestésica), y las preferencias comunicativas (C1: Cinestésica; C2: Visual; C3: Auditiva; C4: Audiovisual) de los grupos; y con el tipo de actividades (D1: Reto; D2: Estructuradas; D3: Experimentación; 
D4: Tiempo) y el trabajo en equipo (E1: Individual; E2: Trabajo por parejas; E3: Pequeños grupos; E4: Gran grupo) que han marcado los estudiantes como preferencia.

Tabla 4. Listado de actividades

\begin{tabular}{|c|c|}
\hline Tipo & No de actividad y Denominación \\
\hline $\begin{array}{l}\text { Actis } \\
\text { inici }\end{array}$ & $\begin{array}{l}\text { 1. Tarea trayectoria personal y profesional; 2.Dinámicas de presen- } \\
\text { tación; 3.Elaboración mapa conceptual; } 4 \text {.Búsqueda de legislación y } \\
\text { comparativa; } 5 \text {.Simulación de un claustro; } 6 \text {. Revisión bibliográfica en } \\
\text { biblioteca. }\end{array}$ \\
\hline $\begin{array}{l}\text { Actividades } \\
\text { de desarrollo }\end{array}$ & $\begin{array}{l}\text { 7. Clase magistral; } 8 \text {.Debate sobre temática actual relacionada con la ma- } \\
\text { teria; } 9 . \text { Búsqueda en internet de material didáctico; 10.Programación de } \\
\text { aula; 11.Actividades en interacción por la facultad (entrevistas); 12.Kahoot; } \\
\text { 13.Twitter. }\end{array}$ \\
\hline $\begin{array}{l}\text { Actividades } \\
\text { de evaluación }\end{array}$ & $\begin{array}{l}\text { 14. Autoevaluación y evaluación del proceso de e-a; 15.Examen individual; } \\
\text { 16.Examen grupal; 17.Vídeo-Exposición; 18.Feria-Exposición; 19.Trabajo } \\
\text { escrito: Viaje a la educación del siglo XXI. }\end{array}$ \\
\hline
\end{tabular}

Tabla 5. Relación de actividades con los aspectos de empoderamiento, estilos de aprendizaje, preferencias comunicativas, tipos de actividades y trabajo en equipo

\begin{tabular}{|c|c|c|c|c|c|c|c|c|c|c|c|c|c|c|c|c|c|c|c|}
\hline & 1 & 2 & 3 & 4 & 5 & 6 & 7 & 8 & 9 & 10 & 11 & 12 & 13 & 14 & 15 & 16 & 17 & 18 & 19 \\
\hline A1 & $\bar{\checkmark}$ & $\bar{x}$ & $\bar{x}$ & $x$ & $x$ & $x$ & $x$ & $\checkmark$ & $\bar{\checkmark}$ & $\checkmark$ & $\checkmark$ & $\checkmark$ & $x$ & $\checkmark$ & $x$ & $\checkmark$ & $\checkmark$ & $\checkmark$ & $x$ \\
\hline $\mathrm{A} 2$ & $x$ & $\checkmark$ & $\checkmark$ & $\checkmark$ & $\checkmark$ & $\checkmark$ & $x$ & $\checkmark$ & $x$ & $\checkmark$ & $\checkmark$ & $\checkmark$ & $\checkmark$ & $\checkmark$ & $x$ & $\checkmark$ & $\checkmark$ & $\checkmark$ & $x$ \\
\hline B1 & $\checkmark$ & $x$ & $\checkmark$ & $\checkmark$ & $\checkmark$ & $\checkmark$ & $x$ & $\checkmark$ & $x$ & $\checkmark$ & $\checkmark$ & $\checkmark$ & $\checkmark$ & $\checkmark$ & $\checkmark$ & $\checkmark$ & $\checkmark$ & $\checkmark$ & $\checkmark$ \\
\hline B2 & $x$ & $\checkmark$ & $\checkmark$ & $\checkmark$ & $\checkmark$ & $\checkmark$ & $x$ & $\checkmark$ & $\checkmark$ & $\checkmark$ & $\checkmark$ & $\checkmark$ & $\checkmark$ & $x$ & $x$ & $\checkmark$ & $\checkmark$ & $\checkmark$ & $\checkmark$ \\
\hline B3 & $\checkmark$ & $x$ & $x$ & $x$ & $x$ & $x$ & $\checkmark$ & $x$ & $\checkmark$ & $\checkmark$ & $x$ & $x$ & $x$ & $\checkmark$ & $\checkmark$ & $x$ & $x$ & $x$ & $x$ \\
\hline B4 & $x$ & $\checkmark$ & $x$ & $x$ & $x$ & $x$ & $x$ & $x$ & $x$ & $x$ & $\checkmark$ & $x$ & $x$ & $x$ & $x$ & $\checkmark$ & $\checkmark$ & $\checkmark$ & $x$ \\
\hline C1 & $x$ & $\checkmark$ & $x$ & $x$ & $x$ & $x$ & $x$ & $x$ & $x$ & $\checkmark$ & $\checkmark$ & $\checkmark$ & $x$ & $x$ & $x$ & $\checkmark$ & $\checkmark$ & $\checkmark$ & $x$ \\
\hline C2 & $\checkmark$ & $x$ & $x$ & $\checkmark$ & $x$ & $\checkmark$ & $\checkmark$ & $x$ & $x$ & $\checkmark$ & $x$ & $\checkmark$ & $\checkmark$ & $\checkmark$ & $\checkmark$ & $\checkmark$ & $\checkmark$ & $\checkmark$ & $x$ \\
\hline C3 & $x$ & $x$ & $x$ & $x$ & $\checkmark$ & $x$ & $\checkmark$ & $\checkmark$ & $x$ & $\checkmark$ & $\checkmark$ & $x$ & $x$ & $x$ & $x$ & $\checkmark$ & $\checkmark$ & $\checkmark$ & $\checkmark$ \\
\hline $\mathrm{C} 4$ & $x$ & $x$ & $\checkmark$ & $x$ & $x$ & $x$ & $\checkmark$ & $x$ & $\checkmark$ & $\checkmark$ & $x$ & $x$ & $\checkmark$ & $x$ & $x$ & $\checkmark$ & $\checkmark$ & $\checkmark$ & $x$ \\
\hline D1 & $x$ & $x$ & $x$ & $\checkmark$ & $x$ & $x$ & $x$ & $x$ & $x$ & $\checkmark$ & $\checkmark$ & $x$ & $x$ & $x$ & $x$ & $\checkmark$ & $\checkmark$ & $\checkmark$ & $x$ \\
\hline D2 & $\checkmark$ & $x$ & $\checkmark$ & $\checkmark$ & $x$ & $\checkmark$ & $x$ & $\checkmark$ & $x$ & $\checkmark$ & $\checkmark$ & $\checkmark$ & $x$ & $\checkmark$ & $\checkmark$ & $\checkmark$ & $x$ & $x$ & $\checkmark$ \\
\hline D3 & $x$ & $\checkmark$ & $x$ & $x$ & $\checkmark$ & $\checkmark$ & $x$ & $x$ & $x$ & $\checkmark$ & $\checkmark$ & $x$ & $\checkmark$ & $x$ & $x$ & $x$ & $\checkmark$ & $\checkmark$ & $x$ \\
\hline D4 & $x$ & $x$ & $x$ & $x$ & $x$ & $x$ & $x$ & $\checkmark$ & $\checkmark$ & $\checkmark$ & $x$ & $x$ & $x$ & $\checkmark$ & $\checkmark$ & $x$ & $\checkmark$ & $\checkmark$ & $\checkmark$ \\
\hline E1 & $\checkmark$ & $x$ & $x$ & $x$ & $x$ & $x$ & $x$ & $x$ & $\checkmark$ & $x$ & $x$ & $x$ & $\checkmark$ & $\checkmark$ & $\checkmark$ & $x$ & $x$ & $x$ & $x$ \\
\hline E2 & $x$ & $x$ & $\checkmark$ & $x$ & $x$ & $x$ & $x$ & $x$ & $\checkmark$ & $x$ & $\checkmark$ & $\checkmark$ & $\checkmark$ & $x$ & $x$ & $x$ & $x$ & $x$ & $x$ \\
\hline E3 & $x$ & $x$ & $\checkmark$ & $\checkmark$ & $x$ & $\checkmark$ & $x$ & $\checkmark$ & $x$ & $\checkmark$ & $\checkmark$ & $\checkmark$ & $x$ & $x$ & $x$ & $\checkmark$ & $\checkmark$ & $\checkmark$ & $\checkmark$ \\
\hline $\mathrm{E} 4$ & $x$ & $\checkmark$ & $\mathrm{x}$ & $x$ & $\checkmark$ & $x$ & $\checkmark$ & $x$ & $x$ & $x$ & $x$ & $\checkmark$ & $x$ & $x$ & $x$ & $x$ & $x$ & $x$ & $x$ \\
\hline
\end{tabular}


d) Determinar el método de evaluación de los alumnos.

Al considerar los diferentes niveles de habilidades del alumnado y aceptar la diversidad de procedimientos metodológicos, la evaluación les va a permitir tomar consciencia de su proceso de aprendizaje. Además de la evaluación final, se ha realizado una evaluación inicial a través de algunas de las preguntas de la tarea de trayectoria personal y profesional, y una evaluación continua al finalizar cada actividad mediante un formulario de google con una serie de preguntas para conocer su progreso durante el proceso de enseñanza aprendizaje (tabla 6).

Tabla 6. Autoevaluación y evaluación del proceso de enseñanza-aprendizaje

\begin{tabular}{|c|c|}
\hline Preguntas & Alternativas de respuesta \\
\hline \multicolumn{2}{|l|}{ Datos personales } \\
\hline $\begin{array}{l}\text { Valoración de las actividades } \\
\text { realizadas }\end{array}$ & $\begin{array}{l}\text { No la he realizado; No me ha aportado nada; Me ha parecido } \\
\text { interesante; Me ha parecido fantástica }\end{array}$ \\
\hline $\begin{array}{l}\text { Señala qué inteligencia has } \\
\text { podido desarrollar }\end{array}$ & $\begin{array}{l}\text { Inteligencias: lingüística; lógico-matemática; visual/espacial; } \\
\text { físico/cinestésica; intrapersonal; interpersonal }\end{array}$ \\
\hline $\begin{array}{l}\text { Señala qué dimensión del } \\
\text { empoderamiento has podido } \\
\text { ejercitar }\end{array}$ & $\begin{array}{l}\text { Autoestima; Autonomía; Capacidad crítica; Eficacia; Identi- } \\
\text { dad comunitaria; Meta-aprendizaje; Participación; Responsa- } \\
\text { bilidad; Trabajo en equipo }\end{array}$ \\
\hline $\begin{array}{l}\text { Señala lo que menos te ha } \\
\text { gustado }\end{array}$ & (Respuesta abierta) \\
\hline $\begin{array}{l}\text { Señala lo que más te ha } \\
\text { gustado }\end{array}$ & (Respuesta abierta) \\
\hline $\begin{array}{l}\text { Si has trabajado en grupo, } \\
\text { señala qué rol o roles has } \\
\text { desempeñado }\end{array}$ & $\begin{array}{l}\text { Investigadora de recursos; Cohesionadora; Coordinadora; Ce- } \\
\text { rebro; Monitora evaluadora; Especialista; Impulsora; Imple- } \\
\text { mentadora; Finalizadora; No he trabajado en equipo }\end{array}$ \\
\hline Valora la clase de hoy & 1: no me ha gustado nada; $5:$ me ha encantado \\
\hline $\begin{array}{l}\text { Expón tu opinión sobre } \\
\text { la presentación de tareas, } \\
\text { desarrollo de las mismas, } \\
\text { aprendizaje... }\end{array}$ & (Respuesta abierta) \\
\hline
\end{tabular}

La referencia sobre los criterios de evaluación de la asignatura se les ha presentado mediante la rúbrica de evaluación de la asignatura (tabla 7) y les sirve como referencia para cualquiera de las actividades evaluables. 
Tabla 7. Rúbrica de evaluación

\begin{tabular}{|c|c|c|c|c|c|c|}
\hline \multicolumn{3}{|c|}{ No logro } & Logro satisfactorio & \multicolumn{2}{|c|}{ Logro notable } & Logro excelente \\
\hline+ & $=$ & - & $=$ & $=$ & - & + \\
\hline $\begin{array}{l}\text { No } \\
\text { ceptc } \\
\text { de la }\end{array}$ & $\begin{array}{l}\text { sub } \\
\text { teria }\end{array}$ & $\begin{array}{l}\text { con- } \\
\text { entes }\end{array}$ & $\begin{array}{l}\text { Conoce desde un } \\
\text { planteamiento teóri- } \\
\text { co fundamentado los } \\
\text { conceptos subyacen- } \\
\text { tes de la materia. }\end{array}$ & $\begin{array}{l}\text { Conoce y } \\
\text { entre sí los c } \\
\text { de la materia } \\
\text { situación } \\
\text { específica. }\end{array}$ & $\begin{array}{l}\text { ciona } \\
\text { eptos } \\
\text { e una } \\
\text { cativa }\end{array}$ & $\begin{array}{l}\text { Posee una visión glo- } \\
\text { balizada de los con- } \\
\text { ceptos subyacentes de } \\
\text { la materia y es capaz } \\
\text { de conectarlos para } \\
\text { proponer propuestas } \\
\text { innovadoras de ma- } \\
\text { nera innovadora }\end{array}$ \\
\hline
\end{tabular}

No todas las actividades que se han desarrollado en el aula han sido evaluables, ya que el objetivo de algunas de ellas era aportar información y conocimiento a las clases magistrales. Las actividades evaluables son las actividades de evaluación que aparecen en la tabla 4.

\section{Conclusión}

Las conclusiones que sobre esta investigación se presentan son parciales en cuanto que muestran una actuación concreta dentro del proyecto de innovación "Los estudiantes como agentes de la educación inclusiva: la elaboración de recursos que faciliten su puesta en práctica" que ha tenido lugar durante el curso 2018-2019. De manera más amplia el proyecto en el que se enmarca este estudio ha generado un impacto en docentes y alumnado de la Facultad de Profesorado y Educación de la Universidad Autónoma de Madrid mediante la difusión y aplicación de acciones que han contribuido a poner en común, difundir y sistematizar la aplicación del currículo multinivel en la Facultad.

En concreto la investigación que nos ocupa ha podido incidir no solo en el currículo multinivel como método inclusivo, sino en un aspecto intrínseco a la educación: la formación de formadores y la coherencia educativa. A partir de una metodología participativa e introspectiva se ha generado un doble alcance: la transmisión del conocimiento de la materia -elaborar programaciones didácticas inclusivas mediante el manejo de las herramientas que aporta el currículo multinivel-; y otra más importante si cabe, referida a la vivencia de una metodología multinivel en el aula universitaria a partir de las individualidades del alumnado. Con la investigación presentada se justifica la necesidad de establecer como reto educativo la atención a la individualidad de cada alumna y cada alumno, y favorecer así una experiencia multinivel vivenciada en el aula.

Los datos de esta investigación clarifican nuestro primer interrogante de investigación afirmando que es posible proporcionar herramientas al alumnado universitario novel de Magisterio para la toma de consciencia sobre la autopercepción de sus 
niveles de empoderamiento, y sus estilos y preferencias de aprendizaje, así como de una toma de consciencia en general de sus experiencias pasadas para la proyección de futuro. Estas herramientas son la tarea de trayectoria personal y profesional, y la tarea de aprendizaje multinivel.

En cuanto a la segunda pregunta de nuestra investigación podemos afirmar que sí se puede diseñar una propuesta pedagógica y curricular de la materia Didáctica General que se adapte a los perfiles individuales y grupales del alumnado del primer curso de Magisterio Infantil y Primaria. Para ello, en primer lugar se debe conocer el perfil individual y grupal y posteriormente diseñar y concretar las actividades que se correspondan atendiendo a un diseño transparente, coherente y participativo.

Todo ello posibilita encontrarnos en el aula con un grupo de estudiantes activos y motivados, donde se les considera agentes de su propio aprendizaje, siendo partícipes en el diseño de su currículo, y capaces de establecer conexiones a partir de sus potencialidades.

Se abre ahora una vía para indagar y profundizar en la aplicación del currículo multinivel en las aulas y valorar su capacidad de transferencia educativa, quizá a través de un estudio longitudinal que nos permitiera conocer la repercusión de los aprendizajes adquiridos en esta asignatura durante sus años universitarios e incluso una vez incorporados laboralmente a un espacio educativo.

\section{REFERENCIAS BIBLIOGRÁFICAS}

Alonso, C. M., Gallego, D. J. y Honey, P. (2010). Cuestionario Honey-Alonso de estilos de aprendizaje; CHAEA. Recuperado de http://www.cua.uam.mx/pdfs/ coplavi/s_p/doc_ng/test-de-estilos-de-aprendizaje.pdf.

Armengol, C. (1999). La cultura organitzacional en els centres educatius de primària. Tesis doctoral. Bellaterra: Universidad Autónoma de Barcelona. Documento inédito.

Arnaiz, P. (1999). Currículum y atención a la diversidad. En M. Verdugo (Coord.). Hacia una nueva concepción de la Discapacidad. Actas de las III Jornadas Científicas de Investigación sobre Personas con Discapacidad.

Belbin, R. M. (2005). Roles de equipo en el trabajo. Bilbao: Belbin Associates.

CAST (2008). Universal design for learning guidelines version 1.0. Wakefield, MA: Author.

Collicott, J. (1991). Implementing Multi-level Instruction: Strategies for Classroom Teachers. En G. L. Porter y D. Richler, (Eds.) Changing Canadian Schools: Perspectives on Disability and Inclusion. Toronto, Ontario: G. Allan Roeher Institute.

Collicott, J. (2000). Posar en pràctica l'ensenyament multinivell: estratègies per als mestres. Suports: Revista catalana d'educació especial i atenció a la diversitat, 4(1). 87-99. Recuperado de: http://www.raco.cat/index.php/Suports/article/ view/102003/141934. 
De la Torre, S. (2009). Transdisciplinariedad y ecoformación: sentir el futuro con otra conciencia. En A. Medina, M. L. Sevillano y S. de la Torre (Coords.), Una universidad para el siglo XXI. Espacio Europeo de Enseñanza Superior (EEES): Una mirada transdisciplinar, ecoformadora e intercultural (pp. 7-16). Madrid: Editorial Universitas.

Díez, M. C. (2009). Las entretelas de la cotidianidad. CEE Participación Educativa, 12, 142-154. Recuperado de http://redined.mecd.gob.es/xmlui/bitstream/ handle/11162/91892/00820113014486.pdf?sequence=1 \&isAllowed=y.

Díez, E. y Sánchez, S. (2015). Diseño universal para el aprendizaje como metodología docente para atender a la diversidad en la universidad. Aula abierta, 43, 8393. http://doi.org/10.1016/j.aula.2014.12.002.

Gardner, H. (1995). Inteligencias múltiples. La teoría en la práctica. Barcelona: Paidós.

Ley 51/2003, de 2 de diciembre, de igualdad de oportunidades, no discriminación y accesibilidad universal de las personas con discapacidad. Boletín Oficial del Estado, $n^{\circ}$ 289, 03 de diciembre de 2003.

Ley Orgánica 6/2001, de 21 de diciembre, de Universidades. Boletín Oficial del Estado, $n^{\circ} 307,24$ de diciembre de 2001.

Morin, E. (2004). La epistemología de la complejidad. Gazeta de Antropología, 20. Recuperado de https://www.ugr.es/ pwlac/G20_02Edgar_Morin.html.

ONU. Informe del Comité de la ONU sobre el estado de la Educación Inclusiva en España. Comité de la Organización de Naciones Unidas, 2018. Recuperado de https://www.ohchr.org/sp/NewsEvents/Pages/DisplayNews. aspx? NewsID=23135\&LangID=S.

Oliver, C. (2009). Algunos retos de la docencia Universitaria actual. En A. Medina, M. L. Sevillano y S. de la Torre (Coords.), Una universidad para el s.XXI. Espacio Europeo de Enseñanza Superior (EEES): Una mirada transdisciplinar, ecoformadora e intercultural (pp. 53-60). Madrid: Editorial Universitas.

Paredes, J., Esteban, R. y Rodrigo-Moriche, M. P. (2019). Didáctica inclusiva y transformadora. El trabajo de los docentes. Madrid: Síntesis.

Planas, A., Trilla, J., Garriga, P., Alonso, A. y Monseny, M. (2016). ¿Qué dimensiones conforman el empoderamiento juvenil? Una propuesta de indicadores. En P. Soler, J. Bellera y A. Planas (Eds.), Pedagogía Social, Juventud y Transformaciones Sociales, (pp. 311-318). Girona: Pedagogía Social, Juventud y Transformaciones Sociales.

Real Decreto 1393/2007, de 29 de octubre, por el que se establece la ordenación de las enseñanzas universitarias oficiales. Boletín Oficial del Estado, $n^{\circ} 260,30$ de octubre de 2007.

Rodrigo-Moriche, M. P. (2018). Diversidad e inclusión. Retos para la convivencia. En Desafíos para la Educación Social en tiempos de cambio. Colectivo JIPS. Málaga: Aljibe. 
Soler, P., Trilla, J., Jiménez-Morales, M. y Úcar, X. (2017). La construcción de un modelo pedagógico del empoderamiento juvenil: espacios, momentos y procesos. Pedagogía Social. Revista Interuniversitaria, 30, 19-34. https://10.7179/ PSRI_2017.30.02Toro (2010).

Toro, J. M. (2005). Educar con “co-razón (8ª ed.). Bilbao: Editorial Desclée de Brouwer.

Úcar, X., Planas, A., Novella, A. y Rodrigo-Moriche, M. P. (2017). Evaluación participativa del empoderamiento juvenil con grupos de jóvenes. Análisis de casos. Pedagogía Social. Revista Interuniversitaria, 30, 67-80. https://doi.org/10.7179/ PSRI_2017.30.05. 